\title{
Assessment of Educational Needs Among Women of Reproductive Age With Common Genital Tract Infections (Vaginitis): The First Step for Developing a Self-care Educational Package
}

\author{
Masoumeh Ebrahimi Tavani ${ }^{1}$, Fazlollah Ghofranipour ${ }^{2}$, Ebrahim Hajizadeh ${ }^{3}$, Mehrandokht Abedini $^{4}$
}

\begin{abstract}
Objectives: Defining the educational needs of women concerning vaginitis and self-care methods aiming at disease treatment is a necessity. This study aimed to determine the educational needs among women at reproductive age with vaginitis in order to design a training program.

Materials and Methods: In a descriptive cross sectional study using purposive sampling, 224 non-pregnant, premenopausal, 15-49 years old, and sexually active females complaining from vaginitis were admitted in the 3 gynecology clinics in Tehran, Iran in 2014. Data on educational needs was collected through a questionnaire containing demographic and disease characteristics and the needs assessment sections, which includes 13 topics of educational needs related to vaginitis based on five score in likert scale.

Results: The mean age of patients was $32 \pm$ (6.9). The average of vaginal infection episodes in the preceding year was 2.8 $\pm(2.6)$. The foremost educational need was sexual self-care of vaginitis. Educational needs averages of the disease information and self-care domains showed a significant statistical difference $(P<.001)$. A total of 115 individuals $(51.4 \%)$ highly needed education based on the total score.

Conclusion: Education is highly needed regarding self-care of vaginitis. The educational need regarding sexual relationship self care is more critical than other topics. The needs in the domain of self care are more important than the domain of disease information. We suggest preparing self-care educational package based on needs assessment as a useful tool to help treatment and prevention of disease recurrence.

Keywords: Educational needs assessment, Genital tract infections, Vaginitis, Self-care, Women
\end{abstract}

\section{Introduction}

The reproductive tract infection (RTI) or genital tract infection (GTI) is a global health problem which affects men, women, families and communities. These infections threat the women's health and may have severe consequences such as infertility, ectopic pregnancy, chronic pelvic pain, abortion and an increased risk of HIV transmission. Therefore, proper prevention and treatment of these diseases are of great importance (1-5).

Vaginitis is the most prevalent infection in genital tract and the most common diagnosis among women (10\%) in primary care section and gynecology clinics. Almost $90 \%$ of the cases are caused by bacterial vaginosis, candidiasis, and trichomoniasis (6-8).

Regarding the increase in prevalence of RTIs and GTIs in various communities, the World Health Organization (WHO) often emphasizes on their prevention and control. The necessity of consultation and education for efficient preventive healthy behaviors is one of the hottest topics in sexual and reproductive health. Education of women at reproduction age towards infection prevention, using health services, and self-care methods aiming at reduction of disease transmission and treatment, is a necessity in societies $(1,9)$.

Nowadays, considering the importance of the self-care in health promotion, lifestyle modification, disease prevention, symptoms evaluation, health protection, treatment and rehabilitation as a complement for health specialized care, the methods for establishment of self-care behaviors are emphasized in disease management (10-12).

Self-care consists of all activities related to health protection, prevention, and treatment, which are conducted by individuals themselves (13). The available evidence suggests that self-care support has its own benefits for public health (14), including reduction of visits required in primary and emergency cares (15), which in turn increase

Received 7 January 2015, Accepted 1 May 2015, Available online 1 October 2015

${ }^{1}$ Ph.D. Candidate, Department of Health Education \& Health Promotion, Faculty of Medical Sciences, Tarbiat Modares University, Tehran, Iran. ${ }^{2}$ Professor, Department of Health Education \& Health Promotion, Faculty of Medical Sciences, Tarbiat Modares University, Tehran, Iran. ${ }^{3}$ Professor, Department of Biostatistics, Faculty of Medical Sciences, Tarbiat Modares University, Tehran, Iran. ${ }^{4}$ Obstetrician \& Gynecologist, Deputy for Public Health, Ministry of Health and Medical Education, Tehran, Iran.

*Corresponding author: Fazlollah Ghofranipour, Email: ghofranf@modares.ac.ir 
patients' satisfaction, life quality, and ultimately promote the individuals' health (14).

Since knowledge and awareness play crucial role in individuals' attitude, behavior and practice, and since favorable practice is possible through acquiring knowledge and changes in attitude, education is of outmost importance. Education of performance skills and helping patients to acquire knowledge, in order to make them follow self-care behaviors, accept and participate in proper health behavior, will result in disease recuperation and complications control $(10,11)$.

The first step of any educational plan is the recognition and prioritization of the educational requirements. Actually, needs assessment is the process of information collection and analysis based on which the needs of individuals, groups, organizations, and communities are recognized and used in curriculum planning $(16,17)$.

Therefore, patients' education must be based on their needs and requirements. Educational need is the gap between what an individual knows and what he or she must know. The gap may be due to lack of knowledge, attitude, and skills (18).

Then ignoring the target group's educational needs would hurt the educational plan's effectiveness (19).

In health education and health promotion plans, the first step for intervention is assessment of health needs. The most successful educational program are based on distinction of the groups at risk, community situation, planning for health education, changing the high risk behaviors, and making the changes stable (20).

Since too many factors affect decision making about a particular health behavior, the health education expert is responsible for determining individuals' personal characteristics, environment and behavior, and then for necessary planning to promote health situation (21).

This study aimed to determine the most important topics of educational needs among women at reproductive age suffering from vaginitis in order to design a training program.

\section{Materials and Methods}

This is a descriptive cross-sectional study conducted using purposive sampling method, in which educational needs of the GTI infected in women at reproduction age were assessed in 3 selected centers in Tehran (Gynecology Clinic of Lolagar hospital, Gynecology boarding clinic of Ibn-e-Sina hospital, and an office of a gynecologist) from January to June 2014.

The study population included all non-pregnant premenopausal, 15-49 year-old females with sexual activity complaining from vaginitis admitted in the determined research centers at the specified period. The sample size included 230 individuals. Inclusion criteria were willingness to participate, informed consent, age between 15-49 years, being sexually active, and being able to read and write.

Six subjects were excluded due to lack of inclusion criteria, having no participation tendency, partial completion of the questionnaire, and non-vaginitis diagnosis during clinical examinations. Finally, 224 questionnaires were considered.

Data collection was conducted using a research-made questionnaire. The researcher reviewed the domestic and foreign literature on related topics and conducted quasi-structural interviews with over 30 patients and then prepared the questionnaire for assessment of educational needs topics. The first section of the questionnaire consisted of demographic characteristics including age, marital status, educations level, occupation, economic status and family income, and health insurance status. The second part has been devoted to disease history including symptoms and patient's complaints, disease records during the preceding year, and the frequency of referrals for treatment. The third part of the questionnaire consisted of 13 topics about assessment of the educational needs. Patients were requested to announce their satisfaction for participating in the project by signing an informed form; then they were asked to complete the questionnaire based on the oral and written explanations of the researcher. The patients with lower level of literacy or with probable difficulties in reading (such as having no glasses) completed their questionnaire with the help the researcher.

The questions were rated on the basis of five score in Likert scale from "very low" to "very high" so that choices of very low scored 1, low scored 2, moderate scored 3, high scored 4 , and very high scored 5 . The score rated from zero for no choice, and 13 for very low choices to 65 for very high choices. Hence, the highest possible score would be 65 , which revealed the highest level of educational needs, concerning the titles.

The questionnaire's content validity was confirmed based on the opinions of 10 experts and professors in health education and promotion, epidemiology, gynecology, infectious disease, social medicine, vital statistics, midwifery, and nursing fields.

We used internal consistency and test-retest reliability methods to assess the reliability of scale. The internal consistency with the Cronbach's alpha coefficient was examined in

20 women. Test-retest of the questionnaire with a 2 -week interval indicated an appropriate stability for the educational needs assessment scale on vaginitis self-care behaviors in the same group ( $\alpha=.96$, ICC $=0.71$ and Pearson correlation $=0.9$ ).

The collected data were analyzed using SPSS 21 software. Kolmogorov-Smirnov test and descriptive statistics (frequency and percentage) were applied along with correlation parametric statistical test, analysis of variance (ANOVA) analysis, and $t$ test with $95 \% \mathrm{CI}$.

\section{Results}

A total of 133 (59.4\%) of subjects were recruited from the Gynecology Clinic of Lolagar hospital (public), 59 (26.3\%) subjects were recruited from Gynecology Clinic of Ibn-e-Sina hospital (private), and 32 (14.3\%) subjects were recruited from the private office of an obstetrician 
and gynecologist. The mean of the educational needs in the centers were $4.010 \pm(0.937), 3.95 \pm(0.888)$ and $4.115 \pm$ (0.836), respectively, which shows no significant difference (Table 1).

Table 1 shows that the subjects' mean of age were $32.0 \pm 6.9$. Their age ranged from 18 to 49 years and mostly between 25-34 year olds (53.6\%). The age of the patients' sexual partners ranged from 19-63, with an average of 36.0 \pm 7.9. According to self-reports, 188 subjects (83.9\%) were married and 36 ones were single, divorced, widows and the rest of the subjects had sexual activity and partner (16.1\%). Concerning the subjects' education level, 85 people
(37.9\%) had high school degree. A total of 125 subjects (56.6\%) were housewives and 53\% (43.4\%) were employed (in public or private sections, self-employed, student, etc.). A total of 121 individuals (54\%) assessed their family at sufficient level of socio-economic status and just one individual reported to be $(0.45 \%)$ higher than sufficient. Additionally, 196 patient (87.5\%) were covered by some kind of health insurance including social insurance, health care, military forces insurance, private insurance, and other organizations' insurance.

Participants had infected vaginitis up to 12 times per the preceding year with an average of $2.8 \pm 2.6$ time, and clin-

Table 1. Distribution of Demographic and Disease Characteristics and Their Relations With Mean of Educational Needs $(\mathrm{N}=224)$

\begin{tabular}{|c|c|c|c|}
\hline Demographic and Disease Characteristics & No. (\%) & Mean $\pm(S D)$ & $\begin{array}{l}\text { Relation With the Mean of } \\
\text { Educational Needs Results ( } P \text { Value) }\end{array}$ \\
\hline Age $(y)$ & $224(100)$ & $32 \pm(6.9)$ & .304 \\
\hline Partner's age (husband, fiancé, etc) (y) & $215(100)$ & $36 \pm(7.9)$ & .651 \\
\hline Classification of Patients age (y) & $224(100)$ & & .455 \\
\hline $15-24$ & $29(12.9)$ & $4.19 \pm(0.859)$ & \\
\hline $25-34$ & $120(53.6)$ & $4.00 \pm(0.876)$ & \\
\hline $35-44$ & $62(27.7)$ & $3.89 \pm(1.012)$ & \\
\hline $45 \geq$ & $13(5.8)$ & $4.17 \pm(0.784)$ & \\
\hline Marital status & $224(100)$ & & .371 \\
\hline Married (having husband) & 188 (83.9) & $3.98 \pm(0.932)$ & \\
\hline Others(single, widow, divorced etc., with sexual partner) & $36(16.1)$ & $4.13 \pm(0.774)$ & \\
\hline Education & $224(100)$ & & .161 \\
\hline Under diploma & $34(15.2)$ & $4.14 \pm(0.933)$ & \\
\hline Diploma & 85 (37.9) & $3.84 \pm(0.923)$ & \\
\hline Associate degree & $23(10.3)$ & $4.26 \pm(0.903)$ & \\
\hline Bachelor degree & $59(26.3)$ & $3.99 \pm(0.857)$ & \\
\hline Master degree and upper & $23(10.3)$ & $4.21 \pm(0.900)$ & \\
\hline Occupation & $221(100)$ & & .980 \\
\hline Housewife & $125(56.6)$ & $4.01 \pm(0.929)$ & \\
\hline Employed & $96(43.4)$ & $4.01 \pm(0.882)$ & \\
\hline Self-evaluation of economic status and family income & $224(100)$ & & .640 \\
\hline Insufficient & $31(13.8)$ & $3.89 \pm(0.965)$ & \\
\hline Somewhat sufficient & $121(54)$ & $4.00 \pm(0.949)$ & \\
\hline Sufficient and more ${ }^{a}$ & $72(32.2)$ & $4.07 \pm(0.817)$ & \\
\hline Having health insurance (public, private, and organizations) & $224(100)$ & & .335 \\
\hline Yes & $196(87.5)$ & $4.03 \pm(0.895)$ & \\
\hline No & $28(12.5)$ & $3.85 \pm(1.00)$ & \\
\hline Vaginitis disease status & $224(100)$ & & \\
\hline Number of vaginitis episodes per the preceding year & $194(86.6)$ & $2.8 \pm(2.6)$ & .932 \\
\hline $\begin{array}{l}\text { Number of visits (referrals) for treatment of vaginitis per the } \\
\text { preceding year }\end{array}$ & $197(87.94)$ & $1.7 \pm(1.4)$ & .536 \\
\hline Period of the beginning of vaginitis symptoms at this episode (days) & $179(79.91)$ & $22.7 \pm(25.4)$ & .098 \\
\hline Study fields & $224(100)$ & & .713 \\
\hline Lolagar hospital, gynecologic clinic (public) & $133(59.4)$ & $4.010 \pm(0.937)$ & \\
\hline Ibn-e-Sina hospital \& boarding clinic, gynecologic clinic (private) & $59(26.3)$ & $3.95 \pm(0.888)$ & \\
\hline Obstetrician and gynecologist office (private) & $32(14.3)$ & $4.115 \pm(0.836)$ & \\
\hline
\end{tabular}

a Only one patient has selected more than sufficient option. 
ic referral up to 7 times with an average of $1.7 \pm 1.4$ time during the preceding year. Duration of symptoms and complaints ranged from 1 to 96 days with an average of $22.7 \pm 24.5$ days.

Based on the statistical tests, no demographic characteristics and disease status determinants had meaningful relation with the average educational needs (Table 1).

Table 2 shows the frequency of topics and domains of patient's educational needs about vaginitis disease and related self-care behaviors. The most important educational needs concerned sexual health self-care to improve vaginitis (mean of $4.14 \pm 0.005$ ), followed by genital health self-care to improve vaginitis (mean of $4.10 \pm 0.981$ ). The least critical needs concerned disease, symptoms, complaints and signs of vaginitis (mean of $3.92 \pm 1.041$ ).

Table 3 compares the average of educational needs in the domains of disease information (educational needs topics 1-7) and self-care (topics of 8-13), which shows a signifi- cant statistical difference $(P<.001)$.

Figure 1 shows levels of educational needs based on the total score of 13 topics. There is no very low need (i.e. score below 13) and the score range is 14-65 with the average of $52.1 \pm(11.9)$. Just five individuals $(2.2 \%)$ had low need, 36 individuals (16.5\%) had moderate, 67 individuals had high and 115 individuals (51.4\%) had very high needs.

\section{Discussion}

According to the findings of the research, there appear educational needs concerning the GTIs at all research locations regardless of being public or private. Also there are female educational needs in various age, education, income and occupational groups. Marital status has been efficient in this regard, revealing that both married and single females felt the needs. In Iran, women especially the younger age groups have no access to proper information about GTIs as well as self-care and self-management

Table 2. Frequency of Topics and Domains of Patient's Educational Needs About Vaginitis Disease and Related Self-care Behaviors $(N=224)$

\begin{tabular}{|c|c|c|c|c|c|c|c|c|}
\hline & \multirow[b]{2}{*}{ Educational Needs Topics } & \multicolumn{7}{|c|}{ Level of the Educational Needs } \\
\hline & & $\begin{array}{l}\text { Very High } \\
\text { (5) No. (\%) }\end{array}$ & $\begin{array}{l}\text { High (4) } \\
\text { No. (\%) }\end{array}$ & $\begin{array}{l}\text { Moderate } \\
\text { (3) No. (\%) }\end{array}$ & $\begin{array}{l}\text { Low (2) } \\
\text { No. (\%) }\end{array}$ & $\begin{array}{l}\text { Very Low } \\
\text { (1) No. (\%) }\end{array}$ & $\begin{array}{l}\text { Total No. } \\
\text { (\%) }\end{array}$ & Mean $^{\mathrm{a}} \pm$ SD \\
\hline 1 & $\begin{array}{l}\text { General information related to female genital } \\
\text { tract, healthy vagina and normal and abnormal } \\
\text { vaginal discharge }\end{array}$ & $91(40.63)$ & $56(25)$ & $58(25.89)$ & $17(7.59)$ & $2(0.89)$ & $224(100)$ & $3.79 \pm 1.026$ \\
\hline 2 & $\begin{array}{l}\text { General information related to genital tract } \\
\text { infections and vaginitis disease (definition, } \\
\text { prevalence, etc) }\end{array}$ & $89(39.73)$ & $60(26.79)$ & $54(24.11)$ & $17(7.59)$ & $4(1.79)$ & $224(100)$ & $3.59 \pm 1.051$ \\
\hline 3 & $\begin{array}{l}\text { Types, signs and symptoms of genital tract } \\
\text { infections (vaginitis) }\end{array}$ & $84(37.5)$ & $63(28.13)$ & $58(25.89)$ & $14(6.25)$ & $5(2.23)$ & $224(100)$ & $3.29 \pm 1.041$ \\
\hline 4 & $\begin{array}{l}\text { Behaviors and risk factors causing the vaginitis } \\
\text { disease }\end{array}$ & $92(41.26)$ & $59(26.46)$ & $49(21.97)$ & $19(8.52)$ & $4(1.79)$ & $223(100)$ & $3.79 \pm 1.067$ \\
\hline 5 & $\begin{array}{l}\text { Consequences and problems of the vaginitis } \\
\text { disease }\end{array}$ & 94 (41.96) & $62(27.68)$ & $40(17.86)$ & $24(10.71)$ & $4(1.79)$ & $224(100)$ & $3.79 \pm 1.092$ \\
\hline 6 & $\begin{array}{l}\text { Healthy behaviors and prevention methods of } \\
\text { recurrent vaginitis }\end{array}$ & $99(44.2)$ & $58(25.89)$ & $47(20.98)$ & $14(6.25)$ & $6(2.68)$ & $224(100)$ & $4.03 \pm 1.071$ \\
\hline 7 & $\begin{array}{l}\text { Therapies (pharmacological and non- } \\
\text { pharmacological) for the treatment of vaginal } \\
\text { infection(vaginitis) }\end{array}$ & $95(42.41)$ & $62(27.68)$ & 47 (20.98) & $16(7.14)$ & $4(1.79)$ & $224(100)$ & $4.02 \pm 1.042$ \\
\hline $\begin{array}{l}\text { Educ } \\
\text { vagi }\end{array}$ & $\begin{array}{l}\text { ational needs on general information domain of } \\
\text { itis (topics 1-7) }\end{array}$ & & & & & & $224(100)$ & $3.97 \pm 0.946$ \\
\hline 8 & $\begin{array}{l}\text { Self-care healthy behaviors domains in genital } \\
\text { tract infections (vaginitis) }\end{array}$ & $97(43.3)$ & $64(28.57)$ & $48(21.43)$ & $9(4.02)$ & $6(2.68)$ & $224(100)$ & $4.06 \pm 1.025$ \\
\hline 9 & $\begin{array}{l}\text { Self-care information on life style and personal } \\
\text { health for the treatment of vaginitis }\end{array}$ & 94 (41.96) & $70(31.25)$ & $47(20.98)$ & $9(4.02)$ & $4(1.79)$ & $224(100)$ & $4.08 \pm 0.974$ \\
\hline 10 & $\begin{array}{l}\text { Self-care information related to genital tract } \\
\text { health for the treatment of vaginitis }\end{array}$ & $100(45.05)$ & $62(27.93)$ & $45(20.27)$ & $13(5.86)$ & $2(0.9)$ & $222(100)$ & $4.10 \pm 0.981$ \\
\hline 11 & $\begin{array}{l}\text { Self-care information related to sexual health } \\
\text { for the treatment of vaginitis }\end{array}$ & $110(49.11)$ & $55(24.55)$ & $42(18.75)$ & $15(6.7)$ & $2(0.89)$ & $224(100)$ & $4.14 \pm 0.005$ \\
\hline 12 & $\begin{array}{l}\text { Self-care Information about the use of } \\
\text { prescribed medication and treatment } \\
\text { recommendations for cure of vaginitis }\end{array}$ & $89(39.73)$ & $64(28.57)$ & 45 (20.09) & $23(10.27)$ & $3(1.34)$ & $224(100)$ & $3.95 \pm 1.064$ \\
\hline 13 & $\begin{array}{l}\text { The importance of adherence and application } \\
\text { and consequences of non-adherence of self- } \\
\text { care information and methods in vaginitis }\end{array}$ & $91(40.63)$ & $62(27.68)$ & $49(21.88)$ & $19(8.48)$ & $3(1.34)$ & $224(100)$ & $3.98 \pm 1.044$ \\
\hline & $\begin{array}{l}\text { ational needs on self-care information domain } \\
\text { ginitis (topics 8-13) }\end{array}$ & & & & & & $224(100)$ & $4.04 \pm 0.923$ \\
\hline Ove & all educational needs ( topics 1-13) & & & & & & $224(100)$ & $4.00 \pm 0.908$ \\
\hline
\end{tabular}

${ }^{a}$ The mean has been calculated based on the 5 score in Likert scale (from 1 for very low to 5 for very high). 
Table 3. Comparison of Patient's Educational Needs Domains (General Information and Self-care Behaviors Information) on the Vaginitis Disease $(\mathrm{N}=224)$

\begin{tabular}{|c|c|c|c|c|}
\hline \multirow{2}{*}{ Educational Needs Domains About Vaginitis } & \multirow{2}{*}{ Mean $^{\mathrm{a}} \pm$ SD } & \multirow{2}{*}{$P$ Value } & \multicolumn{2}{|c|}{$95 \% \mathrm{Cl}$} \\
\hline & & & LL & UL \\
\hline Educational needs on general information domain of vaginitis (7 topics) & $3.975 \pm 0.946$ & \multirow{2}{*}{$<.001$} & 3.851 & 4.100 \\
\hline Educational needs on self care information domain of vaginitis (6 topics) & $4.049 \pm 0.923$ & & 3.927 & 4.171 \\
\hline Overall educational needs (13 topics) & $4.009 \pm 0.908$ & & 3.889 & 4.129 \\
\hline
\end{tabular}

a Mean is based on five score in Likert scale from 1 (very low) to 5 (very high).

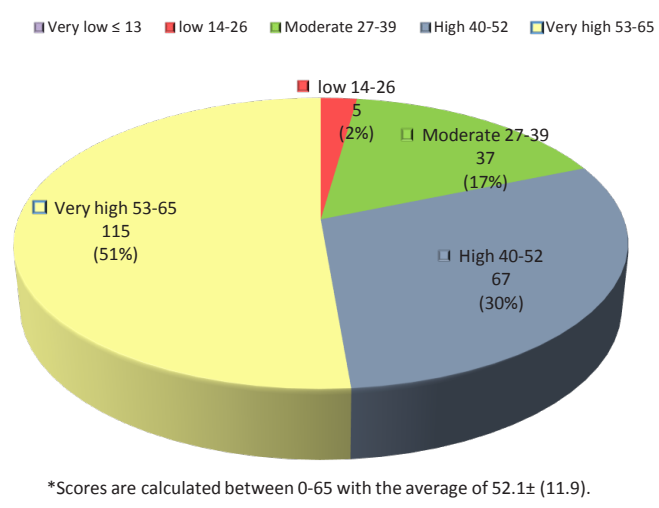

Figure 1. Classification of educational needs levels among reproductive age women with common GTIs (vaginitis) $(\mathrm{N}=224)$

of vaginitis.

Moreover, the subjects' disease status concerning the duration and frequency of infection and referrals had no contribution to the mean of educational needs.

We did not detect any published report on determining the women's educational needs concerning the vaginitis in Iran, but there are a number of studies on GTIs/RTIs and the related factors as well as the related health education. The result of a study under the title of "Women's KAP in prevention of GTI" conducted in Kerman, Iran on 305 women aged 15-49 years, revealed that the subjects achieved $70 \%$ of the total knowledge score, $80 \%$ of the total attitude score, and $80 \%$ of the total practice score; but their educational needs have not been determined (22).

The results of another study on woman's practice and knowledge concerning the genital tract health (at the age of reproduction admitted in Shahid Beheshti hospital, Kashan, Iran) revealed that their knowledge about the subject is at a favorable level. Unfortunately, they had not satisfactory performance. Researchers suggested that women be educated in suitable locations including health centers through educational pamphlets, in order to change their performance (23).

In Iran, studies have confirmed the positive effect of education on improvement of knowledge, attitude and practice in some types of vaginitis. A study in Tehran-Iran has confirmed the positive effects of health education on improvement of knowledge, attitude practice, and duration of fungal vaginitis treatment. Researchers concluded that health education is an efficient method for improving the related variables (24).
Another study under the title of "Knowledge, behaviors and prevalence of GTI: a descriptive study" has been conducted. The findings of this study revealed that GTI prevalence was very high among the low-income rural women and that they were in urgent need for efficient health education proportionate to their culture (25). The results of another research under the title of "Effect of behavioral-educational intervention on woman's knowledge, risk perception and self-efficacy concerning sexually transmitted infections (STIs)" revealed the importance of providing the health care providers with the STIs information, in guiding patients during clinical cares. Women must understand that STIs are greatly related to complications of reproductive health (26).

A study in Sweden was conducted on knowledge about prevention of STDs among the young women with 16-23 years of age. The researchers emphasized on enhancement of knowledge and changing the behavior (27).

Our findings show that the most critical educational need concerned self-care of behaviors in sexual relationships in order to improve vaginitis. In a study on determining the reproductive and sexual needs of couples (Tehran, Iran, 2010), the healthy sexual relationship was specified as the most crucial educational need for both men and women (28).

The results of our study suggest that the least important needs concerned type of diseases, symptoms, complaints and vaginitis signs, because many patients presented with complaints and symptoms after recurrence of the diseases. Although talking about GTIs is usually associated with a feel of shame or stigma, our findings show that educational needs levels have been high and very high and the highest need and interest in education concerned self-care.

Our study has certain limitations as well. Since our culture denies the single, divorced and widow's sexual affairs, the self-report questionnaire was distributed among the patients to be completed if desired. Obviously, some respondents may have introduced themselves as married.

Although, we tried to use different kinds of public and private clinics with various insurance coverage, the limited number of gynecology clinics and centers in the study led to limited sampling locations and our results cannot be generalized to all centers.

The next limitation was related to the diagnosis of vaginitis conducted based on the patients' complaints and clinical and para-clinical (if necessary) criteria in three centers. In two clinic only one gynecologist and in the 
third one at most two specialists with the highest similarity in age, experience, similarity of diagnosis and medicine treatment were responsible for diagnosis of disease with the main researcher.

\section{Conclusion}

The results of the present study showed that the vaginitis infected women in reproductive age need education on GTIs and the related self-cares. Our findings proved that woman's need and interest in education of self-care concerning sexual relationships was more significant than those in other domains. Enhancement of information, motivation, and behavioral skills are necessary to change the related behaviors and to achieve correct self-care behavior.

Regarding our study's results and the self-care program in the Iranian Health Ministry's development plans in the recent years, we suggest the health system and hospitals to prepare self-care and self-management educational package for GTIs or vaginitis, proportionate to the country's culture, It is also suggested that such packages be provided to patients and health care personnel as an important tool to prevent recurrence and to help treatment of the disease. These packages, depending on the patients' desires, may include different educational forms.

Increase in divorce rate in Iran during the recent years as well as extra marital sexual relationships among singles, make it more vital to assess the needs and education of people in the fields of STIs prevention and their complexities.

Due to the acute nature of the vaginitis disease which makes the patients to go to the physician or health care provider as soon as symptoms appear and regarding the fact that many Iranian women are employed and lack enough time, it would be better to assess needs and educate them when they are admitted in clinics and health centers, using the new and modern educational methods. Further studies are suggested on various dimensions of the educational needs of women at the reproductive age with GTIs, specifically on STIs.

\section{Ethical issues}

This paper is extracted from a $\mathrm{PhD}$ thesis of the Health Education and Promotion course approved by the Medical Science Faculty of Tarbiat Modares University, Tehran, Iran in 2014 (No. D52/176-2014) and licensed by Medical Ethics Committee of the university (No. D52/6881-2014).

\section{Financial support}

This research had official support of Tarbiat Modares University but no organizational funding and conducted it using investigator's personal budget.

\section{Conflict of interests}

The authors have no conflicts of interest to disclose.

\section{Acknowledgments}

We appreciate valuable cooperation and helps of digni- fied professors, authorities and staff of Tarbiat Modares University, honorable staff of Lolagar hospital, especially Dr. Tarane Quds and Dr. Lale Mirazaie as well as staff of Ibn-e-Sina Board Clinic and Hospital, in particular the respectable manager and staff of the Gynecology clinics of the 2 centers. We also appreciate the sincere and unwavering assistance of Dr. Mehrandokht Abedini, obstetrician and gynecologist and her honorable office clerk. Finally, we sincerely thank to all participants in this study for their cooperation.

\section{References}

1. World Health Organization. Sexually Transmitted and Other Reproductive Tract Infections Integrating STI/RTI Care for Reproductive Health: A guide to essential practice. Geneva: WHO; 2005.

2. World Health Organization. Sexually Transmitted Infections, The importance of a renewed commitment to STI prevention and control in achieving global sexual and reproductive health. http://apps.who.int/ iris/bitstream/10665/82207/1/WHO_RHR_13.02_ eng.pdf. Published 2013.

3. Population council. Reproductive Tract Infections: an Introductory Overview. http://www.popcouncil.org/ pdfs/RTIFacsheetsRev.pdf. Published 2009.

4. Xia DY, Liao SS, He QY, Choi KH, Mandel JS. Selfreported symptoms of reproductive tract infections among rural women in Hainan, China: prevalence rate and risk factor. Sex Transm Dis. 2004;11(31):643649. doi: 10.1097/01.olq.0000143111.33741.40

5. Msuya SE, Mbizvo E, Stray-Pedersen B, Sundby J, Sam NE, Hussain A. Reproductive tract infections and the risk of HIV among women in Moshi, Tanzania. Acta Obstet Gynecol Scand. 2002;81(9):886-893. doi: 10.1034/j.1600-0412.2002.810916.x

6. Berek JS. Berek \& Novak's Gynecology. 14th ed. Philadelphia: Lippincott Williams \& Wilkins; 2007:854-6.

7. Egan M, Lipsky MS. Diagnosis of vaginitis. Am Fam Physician. 2000; 62:1095-104.

8. Karaer A, Boylu M, Avsar AF. Vaginitis in Turkish women: symptoms, epidemiologic microbiologic association. Eur J Obstet Gynecol Reprod Biol. 2005;121(2): 211-215. doi: 10.1016/j. ejogrb.2004.11.030

9. Ray K, Muralidhar S, Bala M, et al. Comparative study of syndromic and etiological diagnosis of reproductive tract infections/sexually transmitted infections in women in Delhi. Int J Infect Dis. 2009;13(6):e352-359. doi: 10.1016/j.ijid.2008.11.021

10. Rafieefar S, Atarzadeh $M$, Ahmadzad Asl M, et al. Comprehensive program of empowering people to take care of their health. Tehran, Iran: Pazhuheshgaran-e-bedune Marz Institute; 2005.

11. Rafieefar S, Ahmadzad Asl M, Sharifi M, et al. Comprehensive system of health education to patient in Iran. Communication and Health education office, Health Deputy, Ministry of Health and Medical 
Education, Iran, 2005. http://behdasht.gov.ir/ uploads/1_94_29.pdf [Persian].

12. Rafieefar S. From health education to health. Communication and Health education office. Ministry of Health and Medical Education, Iran, $2005 . \quad$ http://www.behdasht.gov. $\mathrm{ir} /$ ? siteid $=1 \&$ pageid $=156 \&$ catid $=64 \&$ dview $=72$ [Persian]

13. Gohar F, Greenfield SM, Beevers DG, Lip GY, Jolly K. Self-care and adherence to medication: a survey in the hypertension outpatient clinic. BMC Complement Altern Med 2008; 8:4. doi: 10.1186/1472-6882-8-4

14. Department of Health. Support for self care in general practice and urgent care settings. 2006. A baseline study. http://webarchive. nationalarchives.gov.uk/20080110005841/http:// dh.gov.uk/en/publicationsandstatistics/publications/ publicationspolicyandguidance/dh_4139969

15. Department ofHealth. Selfcare-A realchoice. Selfcare support- A practical option. 2005. http://webarchive. nationalarchives.gov.uk/20080110005841/http:// dh.gov.uk/en/publicationsandstatistics/publications/ publicationspolicyandguidance/dh_4100717

16. Fathi Vajargah K. Educational Needs Assessment: Models and Techniques. First ed. Fathi Vajargah K, editor. Tehran: Ayyj Publishing House; 2002. [Persian]

17. Mirzabeigi A. Curriculum planning and lesson plan in formal education and human resource training. Tehran: Yastoroon; 2001. [Persian]

18. Mohammadi N, Rafieefar S. Comprehensive curriculum on health education (Introduction to applied program). Tehran: Mehreravash; 2005. [Persian]

19. Morris ML, Carter SA. Transition to marriage: a literature review. Journal of Family and Consumer Sciences Education. 1999;17(1):1-24.

20. McCoy CB, McBride DC. HIV Research with the global context: introduction to special issue. J Urban Health. 2005;82(3): iv2-iv4. doi:10.1093/jurban/jti101
21. Dehdari T, Ramezankhani A, Zarghi A. Principles of Health Promotion. Tehran: Nazari Publication; 2011. [Persian]

22. Farokhzadian J, Mangolian Sharbabaki P. Assessment of Knowledge and Practice about Genital Tract Infections among women referred to Public Health Centers in Kerman (Iran). Fertility and Infertility Journal. 2004;6:346-355. [Persian]

23. Suki Z, Hatamian S. Assessment of Knowledge and Practice about Genital Health among reproductive age women referred to Shahid Beheshti Hospital in Kashan (Iran). Proceeding of the Seminar: Sexual Health in fertility and infertility. 2004. from: http:// www.avicenna.ac.ir/PDF/Pe/SM/10.pdf [Persian]

24. Parsapur R. Effectiveness of Health education on Knowledge, Attitude and Practice (KAP) and Treatment period of women with fungal vaginitis (candidiasis) [MSc Dissertation]. Tehran: Tehran University of Medical Sciences, Public Health Faculty; 2005. [Persian]

25. Li C, Han HR, Lee JE, Lee M, Lee Y, Kim MT. Knowledge, behaviors and prevalence of reproductive tract infections: a descriptive study on rural women in hunchun, china. Asian Nurs Res (Korean Soc Nurs Sci) 2010;4(3):122-129.

26. Johnson-Mallard V. The effects of an education/ behavioral intervention on knowledge, perceived risk and self-efficacy in women. Graduate School Theses and Dissertations. http://scholarcommons.usf.edu/ etd/2944. Published 2005.

27. Andersson-Ellstrom A, Milsom I. Knowledge about the prevention of sexually transmitted diseases: a longitudinal study of young women from 16-23 years of age. Sex Transm Infect. 2002;78(5):339-341.

28. Pourmarzi D, Rimaz S, Merghati Khoei E. Sexual and Reproductive Health Educational Needs in Engaged Couples in Tehran in 2010. Sex Res Soc Policy. 2014;11:225-232. doi: 10.1007/s13178-014-0159-4.

Copyright $\odot 2015$ The Author(s); This is an open-access article distributed under the terms of the Creative Commons Attribution License (http://creativecommons.org/licenses/by/4.0), which permits unrestricted use, distribution, and reproduction in any medium, provided the original work is properly cited. 